33 Urban, L. and Somjen, G. G. (1990) Brain Res. 520, 36-42

34 Cummins, T. R., Jiang, C. and Haddad, G. G. (1993) J. Clin Invest. 91, 608-615

35 Khazipov, R., Bregestovski, P. and Ben-Ari, Y. (1993) J. Neurophysiol. 70, 2251-2259

36 Zhu, P. J and Krnjevic K. (1994) Neurosci Lett $166,27-30$

37 Hershkowitz, H., Katchman, A. N. and Veregge, S. (1993) 1. Neurophysiol. $69,432-441$

38 Rosen, A. 5. and Morris, M. E. (1993) J. Neurophysiol. 69. 109-117

39 Pearson, H. A. and Dolphin. A. C. (1993) Pflügers Arch. 425. 518-527

40 DiPolo, R. and Beaugé, L. (1982) Biochim. Biophys. Acta 688 , 237-245

41 Schubert, P. and Kreutzberg, G. W. (1987) in Topics and Perspectives of Adenosine Research (Gerlach, E. and Becker, B. F., eds), pp. 521-531, Springer-Verlag

42 Hagberg, H. et al. (1987) J. Neurochem. 49, 227-231

43 Phillis, J. W., Walter, G. A. and Simpson, R. E. (1991) J. Neurochem. 56, 644-650

44 Lloyd, H. G. E., Lindstrom, K. and Fredholm, B. B. (1993) Neurochem. Int. 23, 173-185

45 Pedata, F., Latini, S., Pugliese, A. M. and Pepeu, G. (1993) J. Neurochem. 61, 284-289

46 Schubert, P. and Mitzdorf, U. (1979) Brain Res. 172, $186-190$

47 Yoon, K-W. and Rothman, S. M. (1991) J. Neurosci. 11 $1375-1380$

48 Burke, S. P. and Nadler, J. V. (1988) J. Neurochem. 51, $1541-1551$

49 Fredholm, B. B. and Dunwiddie, T. V. (1988) Trends Pharmacol. Sci. 9, 130-134

50 Lloyd, H. G. E., Spence, I. and Johnston, G. A. R. (1988) Brain Res. 462, 391-395
51 Fowler, J. C. (1990) Brain Res. 509, 331-334

52 Gribkoff, V. K., Bauman, L. A. and VanderMaelen, C. P. (1990) Brain Res. 512, 353-357

53 Zhu, P. J. and Krnjevic, K. (1993) Neurosci. Lett. 155 $128-131$

54 Dolphin, A. C., Forda, S. R. and Scott, R. H. (1986) J. Physiol $373,47-61$

55 Fowler, J. C. (1993) Neurosci. Lett. 157, 83-86

56 Andine, P., Orwar, O., Jacobson, I., Sandberg, $M$. and Hagberg, H. (1991) J. Neurochem. 57, 222-229

57 Fabricius, M., Jensen, L. H. and Lauritzen, M. (1993) Brain Res. 612, 61-69

58 Obrenovitch, T. P. et al. (1993) J. Neurochem. 61, 178-186 59 Bosley, T. M. Woodhams, P. L., Gordon, R. D. and Balazs, R. (1983) J. Neurochem. 40, 189-201

60 Burke, S. P. and Nadler, J. V. (1989) Brain Res. 500, 333-342

61 Penning, D. H., Goh, J. W., El-Beheiry, H. and Brien, J. F (1993) Brain Res. 620, 301-304

62 Lloyd, H. G. E., Morrison, M. and Johnston, G. A. R. (1992) Mol. Neuropharmacol. 2, 211-214

63 Drejer, J., Benveniste, H., Diemer, N. H. and Schousboe, A (1985) J. Neurochem. 45, 145-151

64 Katayama, Y. et al. (1991) Brain Res. 558, 136-140

65 Mogul, D. J., Adams, M. E. and Fox, A. P. (1993) Neuron 10 $337-334$

66 Ikeda, M., Nakazawa, T., Abe, K., Kaneko, T. and Yamatsu, K. (1989) Neurosci. Lett. 96, 202-206

67 Bouvier, M., Szatkowski, M., Amato, A. and Attwell, D (1992) Nature 360, 471-474

68 Madl, J. E. and Burgesser, K. (1993) J. Neuroscience 13 4429-4444

69 Choi, D. W. (1990) J. Neuroscience 10, 2493-2501

70 Lee, K. S. and Lowenkopf, T. (1993) Brain Res. 609 $313-315$
Acknowledgements

We thank Garry

Rodda for assistance

with the figures and

Max Bennett

Stephen Redman and

Christian Stricker for

their comments on

the manuscript. The

work was supported

by grants from the

NHand MRC and

Clive and Vera

Ramachiotti

Foundations

\title{
Dendritic processing of synaptic information by sensory interneurons
}

\author{
Alexander Borst and Martin Egelhaaf
}

\begin{abstract}
One of the most distinguishing features of nerve cells is the vast morphological diversity of their input regions, that is, their dendrites. These range from bulbous structures, with only small protrusions, to large treelike arborizations. The diversity of nerve cells is further augmented by a continuously increasing number of types of voltage-dependent conductances in dendrites that might alter the postsynaptic signals in a pronounced way. Moreover, intracellular factors such as $\mathrm{Ca}^{2+}$ link electrical activity with biochemical processes, and can induce short and long-term changes in responsiveness. This complexity of neurons in general, and the uniqueness of each cell type, sharply contrasts with the comparatively simple and uniform design principle of the integrate-and-fire units of so-called neuronal net models. This raises the question of which particular structural and physiological details of nerve cells really matter for the performance of neuronal circuits. An answer to this basic problem of computational neurobiology might be given only if the task of the neurons and circuits is known. This review illustrates how the problem can be approached particularly well in sensory intemeurons. The functional significance of sensory interneurons can often be assessed more easily than that of central nerve cells because of their vicinity to the sensory surface.
\end{abstract}

In general, the signals that are carried by a nerve cell are determined by several factors. These include the output of its presynaptic elements, the transmission characteristics of the input synapses, the spatial distribution of the synapses and the geometry of the dendritic tree, and the intrinsic passive and active electrical properties of the neuron itself. To understand dendritic information processing, all these details of the subcellular machinery underlying neuronal excitability, and their contribution to the overall response properties of the nerve cell will have to be determined, and their functional significance with respect to information processing of the whole system will have to be explained.

In the different systems that are commonly used to investigate cellular information processing, our knowledge is usually limited to only part of the aforementioned aspects. On the one hand, there are neurons, such as the Purkinje cells of the cerebellum $^{1-3}$ or the pyramidal cells in the CA1 region of the hippocampus ${ }^{4,5}$, where we know in unprecedented detail the ionic mechanisms underlying neuronal excitability in various parts of the nerve cell. However, in these systems the computational task of the cell under investigation is often not yet sufficiently understood. On the other hand, in many nerve cells where the computational task might be more obvious, knowledge about the biophysical details is lacking. Therefore, we don't know at present how these cells process their synaptic input and, thus, acquire their specific properties. As a result of recent research

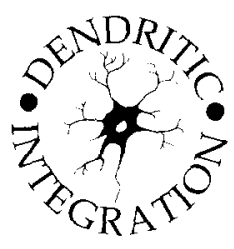

Alexander Borst is at the FriedrichMiescherLaboratorium der Max-PlanckGesellschaft, Spemannstrasse 37 D-72076 Tübingen, Germany, and Martin Egelhaaf is at the Centre for Visual Sciences, Research School of Biological Sciences, Australian National University, GPO BOX 475 Canberra ACT 2601 Australia. 


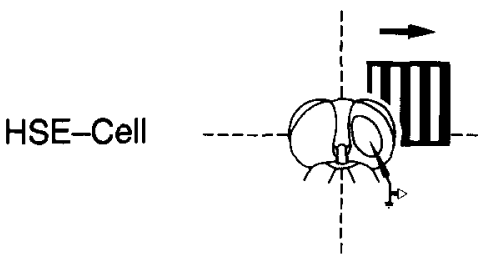

A

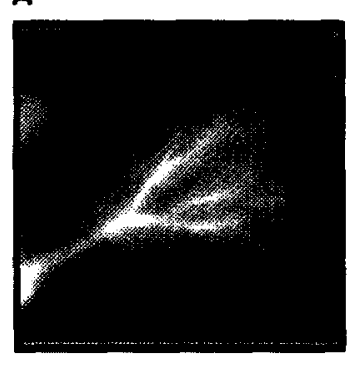

B

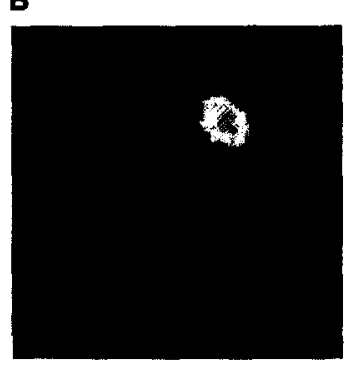

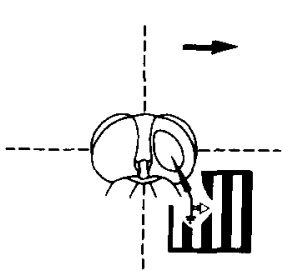

C

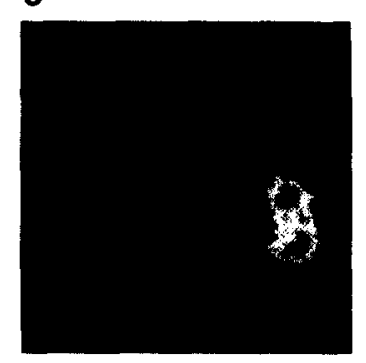

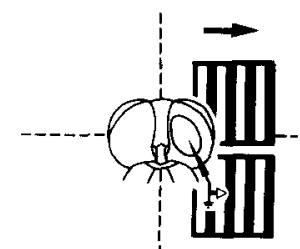

D

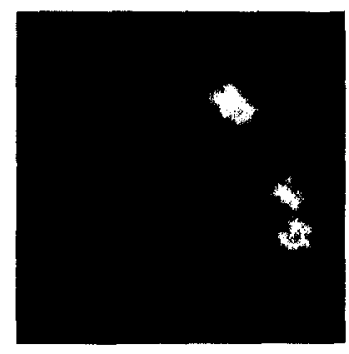

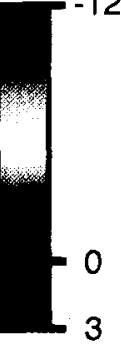

$100 \mu \mathrm{m}$

Fig. 1. Topographic input organization of a visual interneuron. Preferred direction motion (horizontal motion from the front to the back of the fly) in locally restricted areas (visual angle approximately 20\%) in the visual field of the fly Calliphora leads to locally restricted increase in free cytosolic $\mathrm{Ca}^{2+}$ in the dendrites of a motion-sensitive cell (HSE-cell; equatorial cell of the horizontal system). The two stimulus patterns were placed in the receptive field in such a way that they induced membrane-potential changes of about the same amplitude when presented alone. (A) Fluorescence intensity image ( $90 \times 90$ pixels; $100 \mathrm{~ms}$ exposure time) of a HSE-cell filled with fura-2 taken at $380 \mathrm{~nm}$ excitation in the living animal. (B-D) Color-coded changes in the raw relative fluorescence ( $\Delta F / F$, without background subtraction) of the same cell at $380 \mathrm{~nm}$ excitation (90 ×90 pixels; $100 \mathrm{~ms}$ exposure time) after $9 \mathrm{~s}$ of preferred direction motion in the dorsal (B), ventral (C) and both dorsal and ventral (D) parts of the receptive field of the cell. $A$ negative $\Delta F / F$ corresponds to an accumulation of $\mathrm{Ca}^{2+}$. The stimulus conditions are shown schematically on top of each image. Scale bar, $100 \mu \mathrm{m}$. Reproduced, with permission, from Ref. 13.

activities, there are now examples of sensory interneurons that assume an intermediate position between these two extremes. For these examples, a considerable amount of experimental data has been accumulated with respect to (1) the anatomy and physiology of their input circuitry, (2) their response characteristics as elicited by sensory stimulation, and (3) their intrinsic electrical properties. This makes at least an educated guess of their computational tasks possible.

\section{Model predictions}

What computations can be expected to occur in dendrites? How does the distinct structure of the various types of nerve cells relate to their computational performance? One way to address these questions is by modeling the nerve cells ${ }^{6-8}$. The models are usually based on the so-called cable theory of neurons pioneered by Wilfrid Rall in the late $1950 \mathrm{~s}^{9}$ (see also Ref. 10). Here, the nerve cell's anatomy is approximated by a large number of cylinders of variable diameter connected with each other through length resistances. Each of the cylindrical segments is modeled by a membrane compartment consisting, in parallel, of the various ionic membrane conductances and the membrane capacitance. Such models were found to be useful in understanding what type of information processing might be possible by dendrites, and how the dendrite's performance is determined by its characteristic structure and the location of synapses. For example, the following predictions were made $6,7,11,12$.

(1) The postsynaptic signals are weighted according to their electrotonic distance from the axon.

(2) The interaction of EPSPs might be approxi- mated much better by a linear summation if they are spread out on the dendritic tree than if they are electrically close together.

(3) How excitatory and inhibitory synapses interact depends on their relative locations on the dendrite, on the driving forces of the underlying ionic currents, and on the magnitude of the corresponding membrane conductances relative to the leak conductance. If the relative conductance changes are sufficiently small, they are subtracted linearly. In contrast, if the reversal potential of the inhibitory channels is near the resting potential of the cell and the relative conductance changes are large (shunting inhibition) they might interact in a strongly nonlinear way. If shunting inhibition is located on the path between excitation and the axon, it can specifically prevent an excitatory input.

(4) The dendritic structure does not only affect the amplitude of synaptic signals but also their timecourse. In particular, owing to their extended surface area, dendritic spines can slow down the postsynaptic signals and, thus, act as temporal low-pass filters.

According to these considerations, dendrites can be expected to impose severe constraints on the processing of synaptic input signals. However, there are not yet many cases where the functional implications of the dendritic structure for the processing of synaptic information can be demonstrated experimentally.

\section{Topographic projection of the sensory surface onto dendritic trees}

In many sensory neurons, especially visual interneurons, the dendritic morphology reflects the spatial distribution of their input sites, because here the 
sensory pathway is organized in a strictly topographic fashion. The topographic input organization of a visual interneuron has recently been visualized directly using optical recording techniques on the so-called tangential neurons of the third visual neuropile of the fly $^{13,14}$. All of these approximately $50-60$ cells can be identified individually in each animal, owing to their highly invariant structure and response characteristics. They respond selectively to direction of motion in large parts of the visual field, and can be regarded as visual filters for specific types of retinal motion patterns occurring during different flight maneuvers ${ }^{15}$. On synaptic stimulation these cells accumulate $\mathrm{Ca}^{2+}$ in their dendrites ${ }^{13,14}$. Using an intracellular fluorescent indicator of $\mathrm{Ca}^{2+}$, it has been shown that excitatory motion stimuli confined to a locally restricted area within the receptive field of the cell leads to accumulation of $\mathrm{Ca}^{2+}$ in only a small part of its dendrite. The location of this accumulation of $\mathrm{Ca}^{2+}$ varies with the position of the motion stimulus in the outside world (Fig. 1). This illustrates that motion information about the visual world is projected like a two-dimensional image onto the dendritic tree of the visual interneuron.

Based on anatomical findings, a similar kind of topographic projection of the sensory surface onto the dendritic tree is suggested for many types of visual interneurons. In a recent study, the dendritic and receptive fields of directionally selective ganglion cells of the rabbit retina were demonstrated to be congruent ${ }^{16}$. However, in contrast with the fly example, it was not possible to test whether the synaptic input of the ganglion cells is also topographically organized within the confines of the receptive field.

What is the computational consequence of a topographic input organization? It assures that signals originating from neighboring locations in the outside world impinge on neighboring locations of the dendrite. This arrangement enables any operation that should occur only locally (with respect to the input space) to be done with a minimum of expenditure of wiring. In visual interneurons, this operation could be a local amplification mechanism for small postsynaptic signals that would not exceed threshold of voltagegated $\mathrm{Ca}^{2+}$ channels when elicited as a single postsynaptic signal. Whether this is indeed the case, and what the advantage of such an operation would be for visual course control, remains to be seen. On the contrary, if neighboring stimuli provide a strong input signal to the dendrite, the stimuli tend to saturate each other depending on their relative location on the dendrite (see below)

\section{Improved signal-to-noise ratio by pooling of synaptic input}

Another aspect of dendritic processing of synaptic input signals pertains to the stochastic nature of neuronal responses. If the input elements are equivalent from a functional point of view, pooling over many of them corresponds to averaging their activity, which might lead to an increase in the signal-to-noise ratio. This functional consequence of synaptic pooling has been analyzed in the large monopolar cells (LMCs) of the visual system of the fly. Large monopolar cells reside in the first optic neuropile, the lamina, and receive their input from six photorecep-

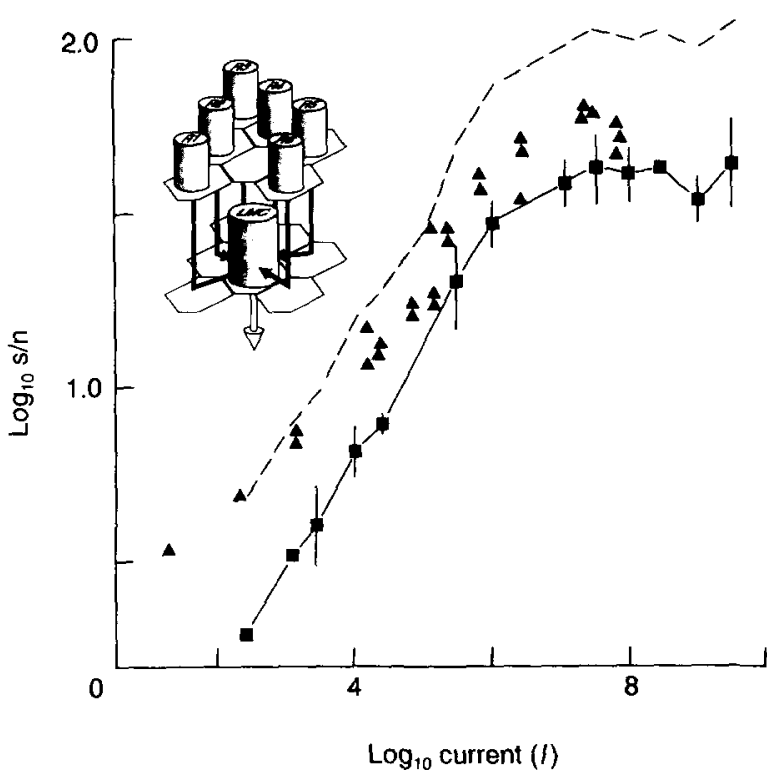

Fig. 2. Improvement of the signal-to-noise ratio $(s / n)$ by synaptic pooling in large monopolar cells (LMCS) of the fly. The inset schematically illustrates that each $L M C$ receives input from six photoreceptors. The signal-tonoise ratio in photoreceptors (filled squares) and LMCS (filled triangles) is measured at different background intensities I. The broken line shows the signal-to-noise ratio expected in $L M C S$ from receptor convergence (improvement by the square root of six). The background intensity 1 is given as the log of the number of effective photons per second per receptor. Reproduced from Ref. 22.

tors that all 'look' at the same small, solid angle of space $^{17,18}$. Each photoreceptor makes over 200 chemical synapses with each LMC (Refs 19-21). Large monopolar cells are essentially cylinders of $2-3 \mu \mathrm{m}$ diameter and $250-1000 \mu \mathrm{m}$ length with only small dendritic protrusions in their postsynaptic segment. When the signal-to-noise ratio of the LMCs is compared with the respective values of the incoming signals from the photoreceptors two types of noise are identified: noise generated in the photoreceptors associated with random photon absorption and phototransduction; and intrinsic noise during transmission of the signals from photoreceptors to LMCs (Ref. 22). Since each photoreceptor contributes an approximately equal number of synapses on the LMCs (Ref. 21 , this convergence, in the absence of intrinsic noise, should improve the signal-to-noise ratio by the square root of six if the responses to the individual photoreceptors add linearly. This improvement is found in the dark-adapted eye, suggesting that under these conditions the performance of the $\mathrm{LMCs}$ is dominated by photon noise (Fig. 2). In contrast, in daylight conditions, their performance is not improved as much and, thus, appears to be limited by synaptic noise 22 .

An improved signal-to-noise ratio has also been implicated to be an essential computational consequence of synaptic pooling by the dendritic tree in ganglion cells of the vertebrate retina. However, the inputs are not equally weighted in these ganglion cells. For example, ON alpha ganglion cells of the cat show a dome-shaped spatial sensitivity distribution ${ }^{23}$. An improved signal-to-noise ratio can be accomplished in at least two ways, either by exploiting that distal dendritic branches are electrotonically further away 


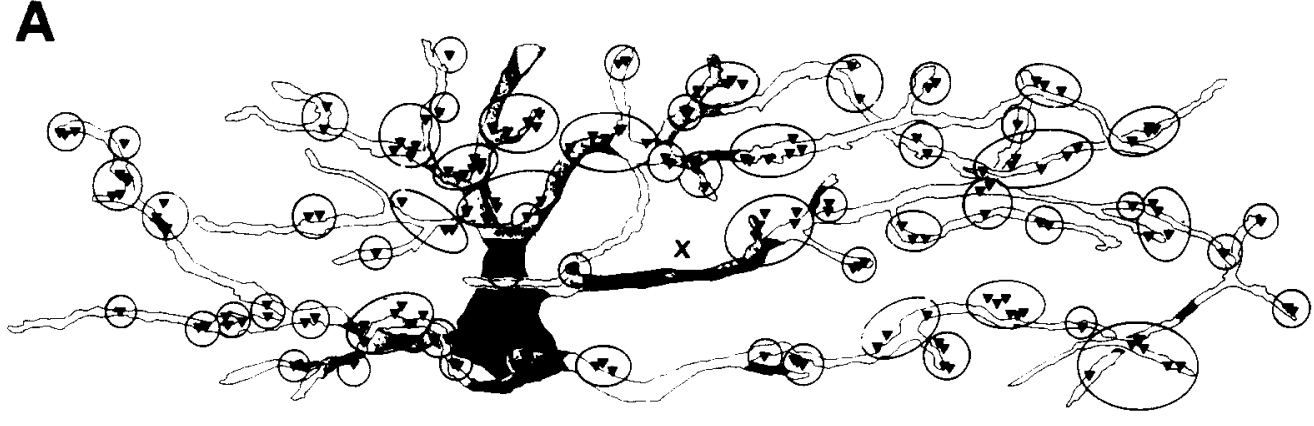

B
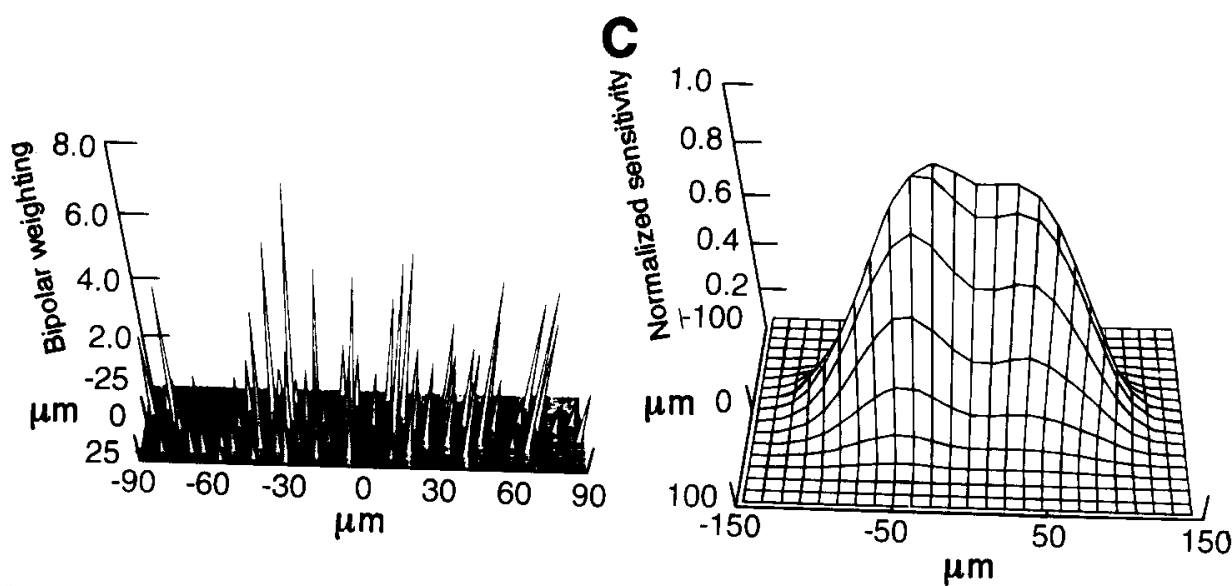

Fig. 3. Spatial sensitivity profile of cat $O N$ alpha ganglion cells is due to the density of input synapses. (A) ON alpha ganglion cell of cat retina reconstructed from serial electron micrograph contacts are from a distribution of contacts the cell receives from b, -bipolar cells. Each set of circled is quantified by determining the weight (nced, with permission, from Ref. 24. (B) This distribution of $62 b_{1}$-bipolar cells that contact an $O$ (needle height) and position (needle location relative to $X$ ) calculated as the product of the number of synapses it cell. For each bipolar cell, its weight was weight it had on the soma, as determined from current injection on the dendrite and its electrotonic ganglion cell at the respective location. (C) current injection into a compartmental model of the centers using weighting in $B$ leads to a from Ref. 23.

in the visual scene. Therefore, depending on the strength of their autocorrelation, the signal-to-noise ratio should improve when partially correlated signals are weighted and summed 25 . In natural scenes, this autocorrelation was shown, on average, to be nearly exponential ${ }^{26}$, thus rendering the dome-like spatial sensitivity of the ganglion cells optimal for improving their signalto-noise ratio.

\section{Directional tuning by spatial weighting of synaptic input}

In the terminal abdominal ganglion of the cricket there is a system of identified interneurons that display directional sensitivity to wind stimuli (Fig. 4). One of these cells (10-3) has three dendritic branches arborizing in different regions of the ganglion (Fig. 4A). At these dendritic branches, they receive their excitatory input from a large number of topographically arranged filiform hairs located on two appendages at the abdomen. By selectively stimulating small patches of these hairs, and photoinactivating individual dendritic branches of the interneuron, it could be shown that the position of a dendritic branch with respect to the topography of the afferent map determines the types of excitatory input the cell can receive (Fig. 4B). In agreement with predictions based

from the axon than the more proximal ones, or by adjusting the density of synapses in the different parts of the dendritic tree accordingly. Compartmental modeling, based on a reconstruction from electronmicrographs of serial sections (Fig. $3 A$ ), shows that for a wide range of specific membrane resistances and linear synaptic transmission characteristics, inputs are almost equally effective at all points on the ON-alphaganglion-cell dendritic tree. Hence, the electrotonic properties of the dendrite appear to contribute only little to the characteristic dome-shaped spatial sensitivity distribution of the excitatory center of the receptive field. Rather, the sensitivity distribution can be accounted for by the dome-shaped density of bipolar cell synapses across the dendritic field of the $\mathrm{ON}$ alpha receptive field ${ }^{23,24}$ (Figs $3 \mathrm{~B}$ and $\mathrm{C}$ ). Model calculations show that the dome-shaped weighting of cone signals optimally improves the signal-to-noise ratio $^{25}$ : if the signals in neighboring cones were statistically equivalent, and if the ganglion cell collected them with equal weight, the signal-to-noise ratio in the ganglion cell should improve by the square root of the number of cones. However, in contrast with the photoreceptor input of the insect LMCs, the input signals to ganglion cells are not identical in a statistical sense but are correlated in a graded way. This correlation decreases with the distance of the input elements in visual space, and is the consequence of optical blurring as well as of spatial autocorrelation on cable theory, the electrotonic distance between the spike-initiating zone of the cell and the various postsynaptic sites determines the relative weighting of each dendritic branch and, thus, plays a decisive role in shaping direction selectivity of the cell ${ }^{27.2 x}$. The synaptic integration of topographically arranged hair sensors is an important computational step in mediating appropriately directed escape responses of the animal ${ }^{28}$.

\section{Temporal filtering by dendritic spines}

Electrosensory neurons in the midbrain of the weakly electric fish Eigenmannia vary considerably in their dendritic structure (Figs $5 \mathrm{~A}$ and $\mathrm{C}$ ). A possible relation between these properties could be determined by recording intracellularly the cell's activity and staining individual neurons, while the electricorgan discharges were mimicked by sinusoidal current sources $^{29}$. Superposition of two such signals with similar frequency leads to amplitude modulations of the carrier frequency ('beats'). Heavily spined neurons respond well to these amplitude modulations as long as their frequency is not too high. However, the response amplitudes decrease markedly as the stimulus frequency is increased (Fig. 5B). In contrast, in aspiny or sparsely spined neurons the stimulusrelated depolarizations do not decrease with increasing modulation frequencies over the range tested (up to $20 \mathrm{~Hz}$ ) (Fig. 5D). These data suggest 
that there is a close correlation between the structure of the dendritic tree and its dynamical response properties: spiny neurons appear to act as low-pass filters of temporal information in sensory signals, whereas aspiny neurons can also code for higher temporal frequencies. This corroborates the hypothesis that dendritic spines contribute to the frequency filtering of synaptic input ${ }^{29}$. The spiny electrosensory neurons of Eigenmannia with their low-pass filter characteristics could play a role as sensory filters to detect the beat rates that are induced by the superposition of the periodic highfrequency discharges of the fish itself and of another neighboring fish that discharges at a similar frequency ${ }^{29}$. Beat rates in the frequency range in which the spiny cells respond optimally are most detrimental to the fish's electrolocation ability. Therefore, upon detection of such beats, the fish either increases or decreases the discharge frequency of its electric organ in order to avoid jamming (jamming avoidance response).
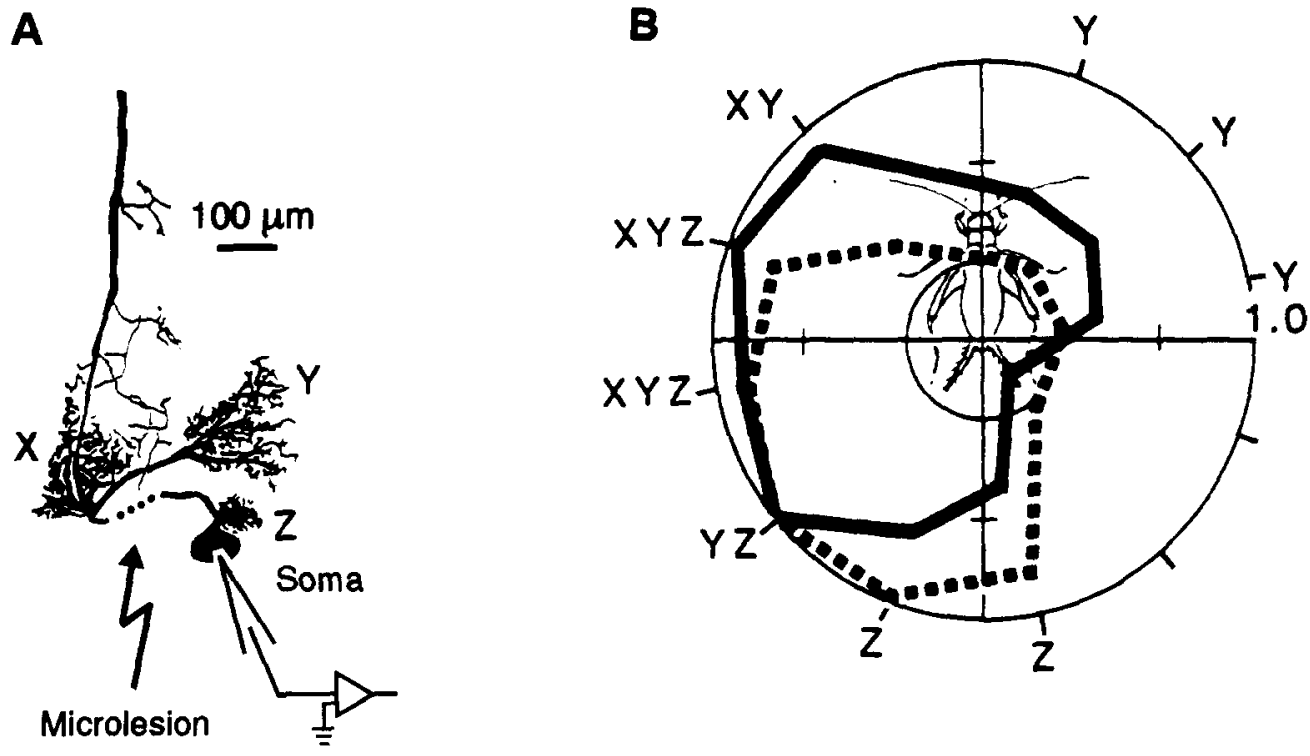

Fig. 4. (A) Wind-sensitive interneuron in the abdominal ganglion of the cricket Acheta with three distinct dendritic regions ( $X, Y$ and $Z$ ) where it receives input from three classes of primary mechanoreceptors sensitive to wind from different directions. The recordings are made from the soma while the cell is filled with a fluorescent dye. After determining the directional tuning of the intact cell, a laser beam is used to inactivate the connection between dendritic region $Z$ near the soma and the rest of the neuron. (B) Directional tuning before (solid line) and after (broken line) laser illumination, both scaled to their own maximum value. The letters around the perimeter of the graph indicate which of the three dendrites should be activated by a wind stimulus from that direction. After photo-inactivation, the directional sensitivity is shifted towards the inputs specific for dendrite Z. Reproduced, with permission, from Ref. 27.

\section{Influence of dendritic topology}

The strictly topographic input organization of motion-sensitive fly interneurons enables visual stimulation of selected dendritic branches using small stimulus patterns confined to localized parts of the cell's receptive field. By combining two excitatory stimuli, that is two stimulus patterns each moving in the cell's preferred direction, it can be shown that the postsynaptic signals superimpose almost linearly if they are elicited in distant branches of the dendritic tree; they saturate each other if they are located close together. Hence, in accordance with predictions derived from cable theory (see above), the extent by which excitatory input signals interact nonlinearly depends on their electrotonic distance ${ }^{30}$. Consequently, cells having the same type of input, but different dendritic geometry, should reveal quite distinct spatial integration properties. This has been discovered recently in the fly when the strength of nonlinear interaction between postsynaptic potentials was compared in cells with palm-like and comb-like dendritic topologies (M. Egelhaaf, J. Haag and A. Borst, unpublished observations). The results emphasize the impact of dendritic topology on dendritic information processing.

\section{Inhibition can be subtractive, relative or absolute}

Although a linear subtraction is a simple operation from a mathematical point of view, to be implemented by neuronal hardware is by no means elementary. An example for an almost linear subtraction is found in the motion detection system of the fly. Motion detection is the primary process of motion computation and takes place in parallel by arrays of retinotopically arranged local movement detectors ${ }^{31}$. In the first processing step, signals originating from neighboring photoreceptors interact in a nonlinear way leading to elements that are directionally selective for motion, at
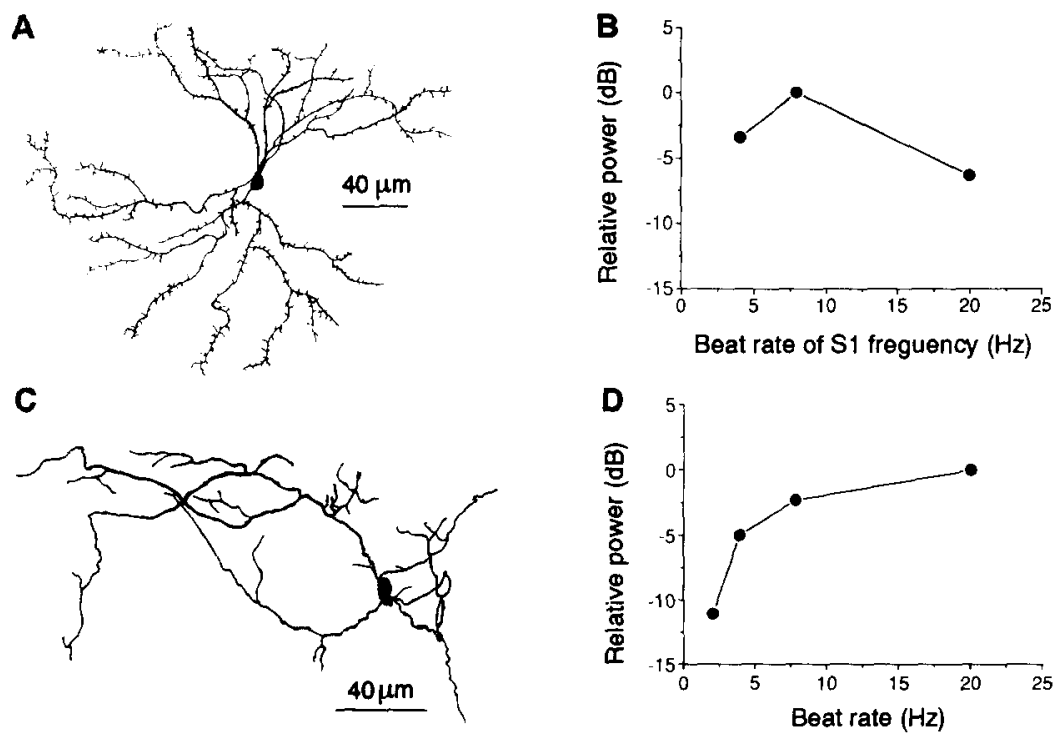

Fig. 5. Frequency filtering by dendrites. Camera lucida drawing from a spiny (A) and an aspiny (B) neuron from layer 5 of the torus semicircularis from the weakly electric fish Eigenmannia. The neurons were recorded intracellularly, and their response to two superimposed sinusoidal current sources of different frequencies ('beat patterns') was determined. These graded potentials were Fourier analyzed after occasional spikes were removed digitally. (C and D) The height of the peak in the power spectrum at the frequency of the stimulus is plotted as a function of the beat frequency for both types of neurons. Note that spiny neurons display a low-pass characteristic while aspiny neurons do not. Reproduced, with permission, from Ref. 29. 
Proximal inhibition

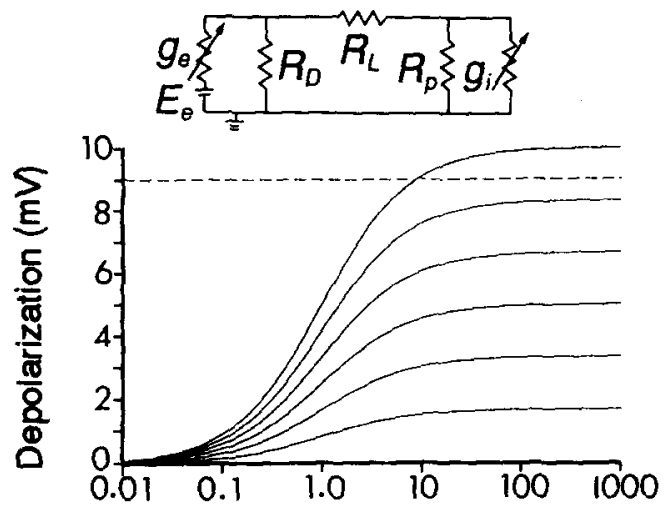

\section{Excitatory strength}

B

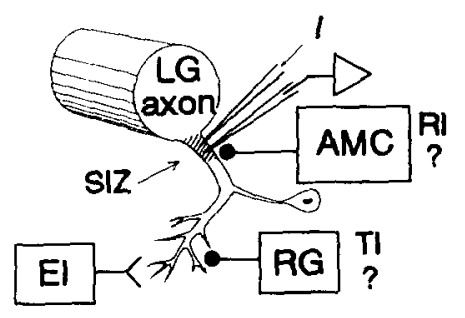

Distal inhibition

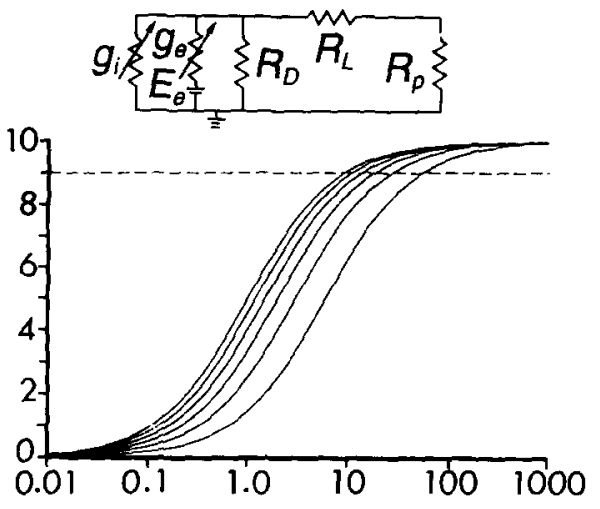

C

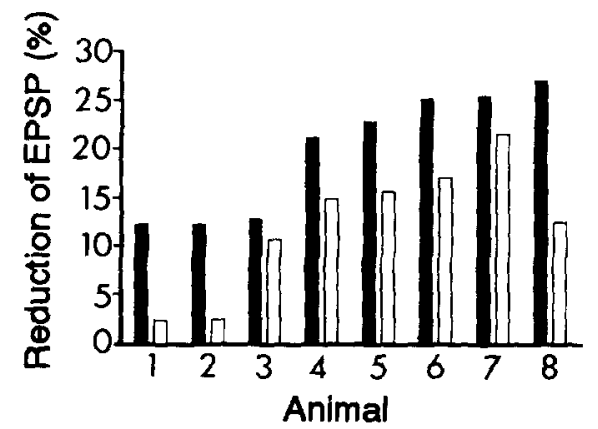

Fig. 6. Computational distinction between proximal and distal inhibition. (A) The circuits differ in the relative placement of the inhibitory $\left(\mathrm{g}_{i}\right)$ and the excitatory $\left(\mathrm{g}_{e}\right)$ conductance. The membrane resistances of the proximal and distal compartment, respectively, are $R_{P}$ and $R_{D}$, the longitudinal resistance is $R_{1}$, the excitatory driving potential is $\mathrm{E}_{e}$. The curves below the circuits were generated with increasing values of $\mathrm{g}_{i} \times \mathrm{R}_{D}$ (O for the top curve, $0.2,0.5,1,2$ and 5 for successively lower curves). Excitation is modeled by the variable excitatory conductance $g_{e}$ and the driving potential $E_{e}=100 \mathrm{mV}$. The resistances are such that $R_{p} /\left(R_{p}+R_{1}\right)=0.1$ and $R_{p}=R_{D}$. Abscissa values given as $\mathrm{g}_{e} \times \mathrm{R}_{D}$. (B) Sensory circuitry excites the lateral giant ( $\left.L G\right)$ dendrites distantly from the spikeinitiating zone $(S I Z$, hatched), where microelectrodes are placed $(I=$ injected current). Shown are the postulated input sites for recurrent $(R I)$ and tonic (TI) inhibition located at proximal and distal places on the dendrites, respectively. Abbreviations: AMC, abdominal motor circuits; El, excitatory input; and RG, rostral ganglia. (C) Attenuation of small (solid bars) and large (open bars) EPSPs by tonic (that is distal) inhibition in eight different animals. As is predicted for distal inhibition (see A), smaller EPSPS are always inhibited more strongly. Reproduced, with permission, from Ref. 34.

least to some extent. In a second processing step, direction selectivity is increased considerably by subtracting the output signals of two such elements with opposite preferred directions. Direction selectivity is optimal if such mirror-symmetrical elements are subtracted with the same weight in a mathematically perfect way ${ }^{32}$. In neuronal terms, this can only be accomplished by the interaction between excitatory and inhibitory synapses, if the driving forces of the underlying ionic currents are equal and the conductance changes are sufficiently small. However, this does not usually occur in most nerve cells where the resting potential is much closer to the reversal potential of the inhibitory channels than of the excitatory channels. This is different in the large tangential cells in the third visual neuropile of the fly. These cells have a comparatively low resting potential of approximately $-50 \mathrm{mV}$ and, thus, almost the same driving forces for excitatory and inhibitory ionic currents. As a consequence, the cells are depolarized and hyperpolarized by approximately the same magni- tude during motion in opposite directions. Hence, a subtractionlike operation takes place on their extended dendritic trees ${ }^{33}$. Owing only to a specific neuronal design, the motion detection system of the fly achieves a direction selectivity close to the optimum that can be achieved in a mathematically perfect system.

When solving other computational tasks, however, the inhibitory synapses are required to interact with the excitatory synapses in a nonlinear way. An example of this, as concluded by combining experimental and theoretical analysis, is found in the neural circuit controling escape behavior of the crayfish ${ }^{34}$. The escape response is mediated by the socalled lateral giant (LG) command neurons. Both the behavioral response and the activity of these neurons can be suppressed in two ways: initiation of a new escape response is completely suppressed while an escape response is just in progress; and when crayfish are feeding, for example, an escape response is not completely suppressed but can be elicited depending on the relative strength of the inhibitory drive. In the $L G$ command neurons, both forms of suppression of excitatory input can be found and correlated with the location of the respective inhibitory synapses on the dendritic tree (Fig. 6). Inhibition on proximal dendrites completely attenuates excitatory input, so that no amount of excitation causes firing. The escape response is, thus, safely suppressed. In contrast, inhibitory synapses on distal dendritic branches inhibit to a much lesser extent. Therefore, any amount of inhibition can be overcome by sufficient excitation. Consequently, the threshold for escape behavior is just shifted under these conditions. Again, the specific design of the spatial distribution of synapses on the dendritic tree might lead to a quite complex performance of single neurons, and even of a whole animal.

\section{Concluding remarks}

In this review, we considered how dendritic processing of synaptic input in sensory interneurons of vertebrates and invertebrates might accomplish a wide range of different computations. These computations comprise temporal frequency filtering by dendritic spines, improvement of the signal-to-noise ratio by adjusting the spatial sensitivity profile of a neuron to the statistical structure of the visual environment and the achievement of a particular directional tuning curve by appropriately weighting the synaptic input. Such diverse computational tasks can be solved at the level of dendritic information 
processing of single neurons, because all neurons appear to be specially adapted to their tasks by means of the structure of their dendritic trees, the distribution of their input synapses and their electrical characteristics. In this respect, all of the cells or cell types that have been discussed here are unique and, thus, differ from the uniform design principle of the usual integrate-and-fire units that make up most connectionist models.

It should be noted that all the dendritic computations discussed above can be accounted for on the basis of a dendrite without voltage-gated ion channels. However, given the evidence for active processes in the dendrites of many neurons, this is likely to be a much too simplistic view ${ }^{35}$. The properties of dendritic voltage-gated ion channels have not yet been determined in all the systems discussed here, but even where they are known their computational significance is not yet clear. In any case, even with passive dendrites, nerve cells can behave as rather complex computing devices. It appears that much of the specificity of neuronal computations relies on the specific anatomical and biophysical design of the neurons that are involved in a task, rather than just in the wiring of uniform neural elements.

\section{Selected references}

$\uparrow$ Llinas, R. and Sugimori, M. (1980) J. Physiol. 305, 171-195

2 Lev-Ram, V., Miyakawa, H., Lasser-Ross, N. and Ross, W. N. (1992) J. Neurophysiol. 68, 1167-1177

3 Miyakawa, H., Lev-Ram, V., Lasser-Ross, N. and Ross, W. N. (1992) J. Neurophysiol. 68, 1178-1189

4 Regehr, W. C. and Tank, D. W. (1992) J. Neurosci. 12, $4202-4223$

5 Miyakawa, H. et al. (1992) Neuron 9, 1163-1173

6 Rall, W. (1989) in Methods in Neuronal Modeling: From Synapses to Networks (Koch, C. and Segev, I., eds), pp. 9-62, MIT Press

7 Segev, I., Fleshman, J. W. and Burke, R. E. (1989) in Methods in Neuronal Modeling: From Synapses to Networks (Koch, C. and Segev, I., eds), pp. 63-96, MIT Press
8 Segev, I. (1992) Trends Neurosci. 15, 414-421

9 Rall, W. (1957) Science 126, 454

10 Rall, W., Burke, R. E., Smith, T. G., Nelson, P. G. and Frank, K. (1967) J. Neurophysiol. 30, 1169-1193

11 Koch, C., Poggio, T. and Torre, V. (1982) Philos. Trans. R. Soc. London 298, 227-264

12 Koch, C. and Poggio, T. (1983) Proc. R. Soc. London, Ser. B $218,455-477$

13 Borst, A. and Egelhaaf, M. (1992) Proc. Natl Acad. Sci. USA $89,4139-4143$

14 Egelhaaf, M., Borst, A., Warzecha, A. K., Flecks, S. and Wildemann, A. (1993) J. Neurophysiol. 69, 340-351

15 Hausen, K. and Egelhaaf, M. (1989) in Facets of Vision (Stavenga, D. G. and Hardie, R. C., eds), pp. 391-424, Springer

16 Yang, G. and Masland, R. H. (1992) Science 258, 1949-1952

17 Kirschfeld, K. (1967) Exp. Brain Res. 3, 248-270

18 Laughlin, S. (1981) in Handbook of Sensory Physiology, Vol. I/ (Autrum, H., ed.), pp. 133-280, Springer

19 Shaw, S. R. (1984) J. Exp. Biol. 112, 225-251

20 Shaw, S. R., Fröhlich, A. and Meinertzhagen, I. A. (1989) Cell Tiss. Res. 257, 295-302

21 Nicol, D. and Meinertzhagen, I. A. (1982) J. Comp. Neurol. 207, 29-44

22 Laughlin, S. B., Howard, J. and Blakeslee, B. (1987) Proc. R. Soc. London, Ser. B 231, 437-467

23 Freed, M. A., Smith, R. G. and Sterling, P. (1992) Proc. Nat/ Acad. SCi. USA 89, 236-240

24 Freed, M. A. and Sterling, P. (1988) J. Neurosci. 8, $2303-2320$

25 Tsukamoto, Y., Smith, R. G. and Sterling, P. (1990) Proc. Natl Acad. Sci. USA 87, 1860-1864

26 van Hateren, J. H. (1992) J. Comp. Physiol. 171, A157-A170

27 Jacobs, G. A. and Miller, J. P. (1985) Science 228, 344-346

28 Jacobs, G. A., Miller, J. P. and Murphey, R. K. (1986) f. Neurosci. 6, 2298-2311

29 Rose, G. J. and Call, S. J. (1993) J. Neurosci. 13, 1178-1189

30 Haag, J., Egelhaaf, M. and Borst, A. (1992) Neurosci. Lett. $140,173-176$

31 Borst, A. and Egelhaaf, M. (1989) Trends Neurosci. 12, 297-306

32 Egelhaaf, M., Borst, A. and Reichardt, W. (1989) J. Opt. Soc. Am. A6, 1070-1087

33 Borst, A. and Egelhaaf, M. (1990) Proc. Natl Acad. Sci. USA 87, 9363-9367

$34 \mathrm{Vu}, \mathrm{E} . \mathrm{T}$. and Krasne, F. B. (1992) Science 255, 1710-1712 35 Llinas, R. R. (1988) Science 242, 1654-1664
A
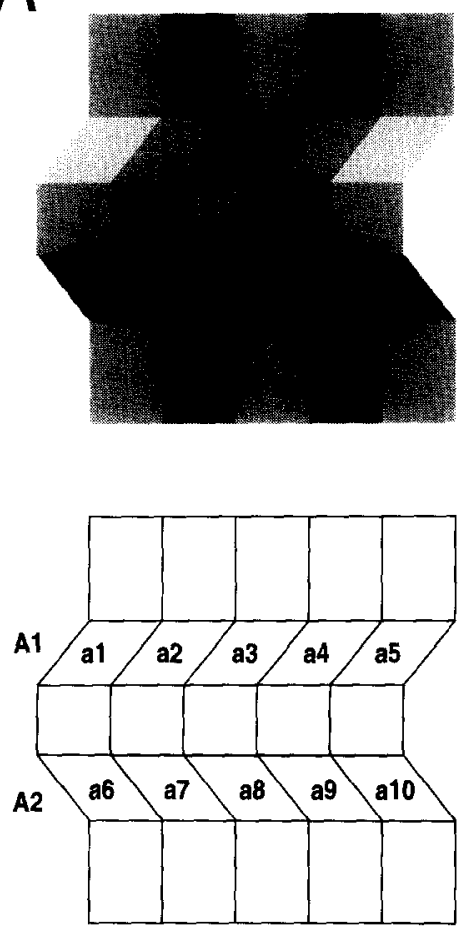

B

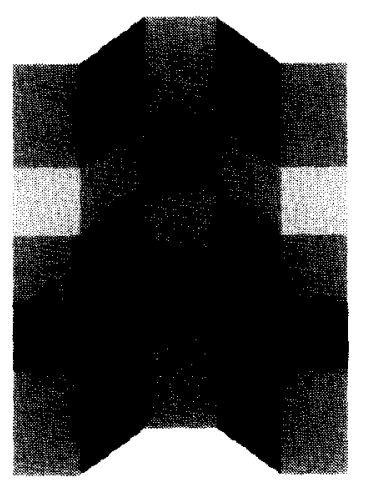

B1

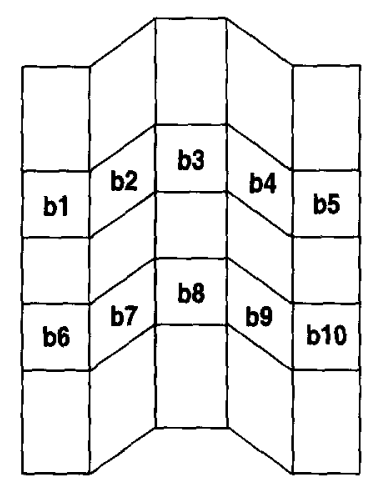

\section{Corrigendum}

In the May issue of TINS (Vol. 17. p. 176), Fig. 2 was reproduced poorly. The correct version is printed here. We apologize to the author and to the readers for this mistake.

Fig. 2. The new 'corrugated plaid' brightness illusion devised by Adelson? (A) Illustrates the dependence of brightness judgements upon highlevel factors, such as perceived depth, form and shading. Patches $a 3$ and $a 8$ are identical, but a 8 appears brighter. (B) The greatly reduced brightness illusion (patches b3 versus b8) associated with a different perceptual interpretation illustrates the inadequacy of low-level explanations. See text for details. Reproduced and modified, with permission, from Ref. 2.
Acknowledgements We are grateful to

T. Brotz, K. C. Cötz,

J. Haag, S. Laughlin,

J. P. Miller and

A. K. Warzecha for carefully reading the manuscript. 